University of Wollongong

Research Online

Faculty of Engineering and Information

Faculty of Engineering and Information

Sciences - Papers: Part A

Sciences

2015

Incorporating robustness in diagonally-relaxed orthogonal projections method for proton computed tomography

\author{
Paniz Karbasi \\ Baylor University \\ Blake Schultze \\ Baylor University \\ Valentina Giacometti \\ University of Wollongong,vg406@uowmail.edu.au \\ Tia E. Plautz \\ University of California, tiaplautz@gmail.com \\ Keith Schubert \\ Baylor University
}

See next page for additional authors

Follow this and additional works at: https://ro.uow.edu.au/eispapers

Part of the Engineering Commons, and the Science and Technology Studies Commons

Research Online is the open access institutional repository for the University of Wollongong. For further information contact the UOW Library: research-pubs@uow.edu.au 


\title{
Incorporating robustness in diagonally-relaxed orthogonal projections method for proton computed tomography
}

\author{
Abstract \\ Iterative algorithms such as ART, DROP, and CARP are commonly used in reconstructing computed \\ tomography images, but only account for errors in the measurements. Errors in the predicted path and \\ intersection lengths, or even blocks of missing measurements can result in degraded image quality. \\ Robust techniques allow for errors in other areas of the model and produce good images that show less \\ sensitivity. In this paper we introduce a robust version of DROP and compare its performance advantages \\ to the standard DROP algorithm on on real data.

\section{Disciplines} \\ Engineering | Science and Technology Studies

\section{Publication Details} \\ Karbasi, P., Schultze, B., Giacometti, V., Plautz, T., Schubert, K. E., Schulte, R. W. \& Bashkirov, V. A. (2015). \\ Incorporating robustness in diagonally-relaxed orthogonal projections method for proton computed \\ tomography. 2015 IEEE Nuclear Science Symposium and Medical Imaging Conference (NSS/MIC) (pp. \\ 1-4). United States: IEEE.

\section{Authors} \\ Paniz Karbasi, Blake Schultze, Valentina Giacometti, Tia E. Plautz, Keith Schubert, Reinhard W. Schulte, \\ and Vladimir Bashkirov
}




\title{
Incorporating Robustness in Diagonally-Relaxed Orthogonal Projections Method for Proton Computed Tomography
}

\author{
Paniz Karbasi ${ }^{[1]}$, Blake Schultze ${ }^{[1]}$, Valentina Giacometti ${ }^{[2],[4]}$, Tia Plautz ${ }^{[3]}$ Member, IEEE, \\ $\underline{\text { Keith E. Schubert }}^{[1],[4]}$ Senior Member, IEEE, Reinhard W. Schulte ${ }^{[4]}$ Member, IEEE, and Vladimir A. Bashkirov ${ }^{[4]}$
}

\begin{abstract}
Iterative algorithms such as ART, DROP, and CARP are commonly used in reconstructing computed tomography images, but only account for errors in the measurements. Errors in the predicted path and intersection lengths, or even blocks of missing measurements can result in degraded image quality. Robust techniques allow for errors in other areas of the model and produce good images that show less sensitivity. In this paper we introduce a robust version of DROP and compare its performance advantages to the standard DROP algorithm on on real data.
\end{abstract}

\section{INTRODUCTION}

Proton computed tomography (pCT) is a novel medical imaging modality developed for treatment planning of proton radiation therapy [1]. In pCT, the goal is to find a good approximation to the linear system of equations of the form $A x=b . A$ is an $m \times n$ sparse matrix such that $a_{j}^{i}$ is the intersection length of the $i$ th proton history with the $j$ th voxel element, and $b_{i}$ is an $m \times 1$ vector that contains the waterequivalent path length (WEPL) measurements, which means that if the proton has the given path-length in water, it will lose the same amount of energy on average. Having this data, one can reconstruct the relative stopping power (RSP) of protons which is the image vector $x$.

Uncertainty due to multiple coulomb scattering and energy straggling are known to be significant challenges [2]. These errors not only effect the WEPL calculations of $b$, they also effect the calculation of the elements of the $A$ matrix. Errors

The research in proton CT is supported by the National Institute of Biomedical Imaging and Bioengineering (NIBIB), and the National Science Foundation (NSF), award Number R01EB013118, and the United States Israel Binational Science Foundation (BSF) grant nos. 2009012 and 2013003. The content of this paper is solely the responsibility of the authors and does not necessarily represent the official views of the National Institute of Biomedical Imaging and Bioengineering or the National Institutes of Health.

[1] Paniz Karbasi, Blake Schultze, and Keith Schubert are with the Department of Electrical and Computer Engineering, Baylor University, Waco, TX 76798 USA, email: Paniz_Karbasi@baylor.edu, Blake_Schultze@baylor.edu, Keith_Schubert@baylor.edu

[2] Valentina Giacometti is with the Center for Medical Radiation Physics, University of Wollongong, Wollongong, NSW, Australia, email: valentina8giacometti@gmail.com

[3] Tia Plautz is with the Santa Cruz Institute for Particle Physics, University of California, Santa Cruz, Santa Cruz, CA 95064, USA, email: tiaplautz@gmail.com

[4] Valentina Giacometti, Keith Schubert, Reinhard Schulte (rschulte@llu.edu), and Vladimir Bashkirov (vbashkirov@dominion.llumc.edu) are with the Department of Basic Sciences, Loma Linda University Medical Center, Loma Linda, CA 92354 USA in the $A$ matrix can result in voxels getting the wrong amount of update, or even the incorrect voxel being updated. The resulting images could have boundaries with artifacts that reflect voxels with the wrong RSP values, or inaccurate RSP values related to the different materials inside the object. In both cases, the accuracy of the results are affected negatively. The goal of this work is to incorporate robust estimation in the diagonally-relaxed orthogonal projections (DROP) algorithm to account for uncertainties in the model and improve the RSP's accuracy. Based on the results of our experiments, the robust iterative solver produces pCT images with less artifacts on the boundary of the object and more accurate RSP values.

There are different reconstruction algorithms for finding the solution of the pCT problem. Diagonally-relaxed orthogonal projections (DROP) is a component averaging technique for solving the linear system $A x=b$. The structure of DROP is outlined in Algorithm 1:

\section{Algorithm 1 (DROP)}

Initialization: choose an arbitrary $x^{0} \in \mathbb{R}^{n}$

Iterative Step: Given $x^{k}$, the next iteration $x^{k+1}$ is

$$
x^{k+1}=x^{k}+\lambda_{k} S^{k} \sum_{i \in I_{t}(k)} \frac{b_{i}-\left\langle a^{i}, x^{k}\right\rangle}{\left\|\left(a^{i}\right)\right\|^{2}} a^{i}
$$

where $S=\operatorname{diag}\left(\frac{1}{s_{j}}\right)$, such that $s_{j}$ is the number of times voxel $j$ is intersected by a path $i \in I_{t}(k)$ [3].

For filling the cells of the $A$ matrix, one needs to find an approximation of the path traversed by each proton through the object. The most likely path (MLP) formalism provides an accurate method for estimating the path of a proton through the object [4].

Regardless of the method that is used for proton path estimation, these paths could have two possible sources of uncertainties: the actual path itself, and the intersection length between the proton and the voxels along its path. Having an accurate estimation of the object hull significantly improves the accuracy of path estimation [5], but these measurements are still inaccurate to some extent, and these uncertainties in our model can contribute to the accuracy of the RSP values negatively, and can cause artifacts and boundary issues in a reconstructed image. Therefore, we need to take these uncertainties into account and re-model the pCT problem. In this paper, we present a robust iterative solver that takes into account the error associated with the intersection length 
between a proton traversing the object and the voxels along its path.

\section{Methodology}

DROP (Algorithm1) generates accurate RSP values when $x_{j} \approx 1$, but when $x_{j} \gg 1$ or $x_{j} \ll 1$, DROP tends to make the value of $x_{j}$ closer to one. In order to prevent DROP forcing voxel values closer to one, we perform the update of $x_{j}$ based on the value of $\left(1-x_{j}\right) \eta$, such that $\left\|E_{A_{i}}\right\| \leq \eta$, where $E_{A_{i}}$ is the error in the $i$ th row of the $A$ matrix. Therefore, we have taken the uncertainty related to the $A$ matrix into account. Based on these facts, we have derived a modified version of Algorithm 1 that is outlined in Algorithm 2.

\section{Algorithm 2}

Initialization: choose an arbitrary $x^{0} \in \mathbb{R}^{n}$ Iterative Step: Given $x^{k}$, the next iteration $x^{k+1}$ is

$$
\begin{gathered}
x^{k+1}=x^{k}+\lambda_{k} S^{k} \sum_{i \in I_{t}(k)} \sum_{j} \frac{b_{i}-\left\langle a^{i}, x^{k}\right\rangle}{\left\|\left(a^{i}\right)\right\|^{2} \pm \psi_{j}^{i}} a^{i} \\
\psi_{j}^{i}=\left(1-x_{j}\right) \eta
\end{gathered}
$$

\section{RESULTS}

In this section, we illustrate the results of reconstructing pCT images with both Algorithms 1 and 2, while we have calculated the mean cord length for each proton history based on the entry and exit angles of the proton [6]. Therefore, for a single proton, the intersection length with the voxels along its path is fixed. The data set used for image reconstruction contained about 120 million proton events generated by a Geant 4 simulated scan of the CTP404 phantom with $4^{\circ}$ increments in projection angle.

In the first experiment, we have used the $A$ matrix resulting from the MLP calculations. In the second experiment, we have added some Gaussian noise with zero mean and $\eta^{2}$ variance to each nonzero element of $A$ as follows:

$$
A_{\text {error }}=A_{M L P}+\eta * N(0,1)
$$

Finally, in the third experiment, we have randomly removed 120000 of the histories from the data set and compared Robust with DROP. This experiment simulates the situation that during a scan, the rate at which protons have been shot towards the object is higher than the processing rate of the recording the histories, thus some of the protons have been missed during the scan. This could lead to an uncertain path matrix, and therefore inaccurate RSPs during the reconstruction.

Figures $1 \mathrm{a}$ and $1 \mathrm{~b}$ illustrate the results of reconstructing images with Algorithms 1 and 2, respectively. Also, Table I includes the reconstructed RSP values by DROP and Robust algorithms.

Figures 2 and 3 illustrate the results of the reconstructed images using Algorithms 1 and 2 when the $A$ matrix contains additive Gaussian noise while $\eta=1.0$ and $\eta=2.0$ respectively. Based on the results in Figures 2 and 3, when using DROP with the noisy path matrix, we have some voxels with RSP values much less than or greater than one on the boundary of the object. Clusters of voxels that have been marked in

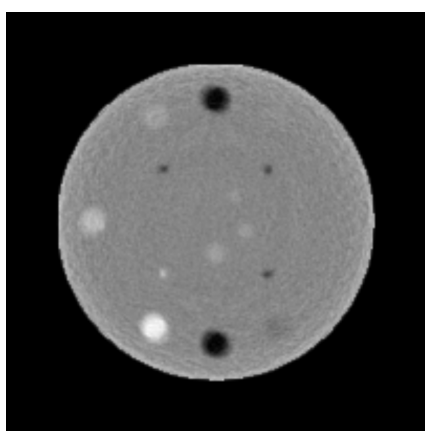

(a) DROP

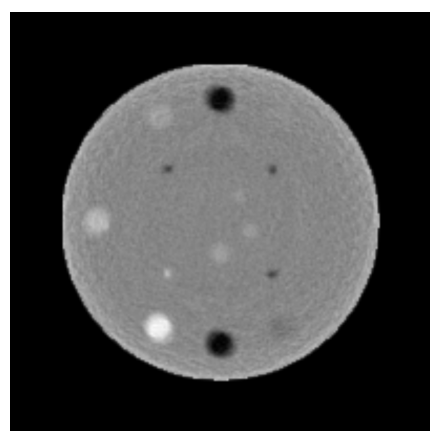

(b) Robust DROP
Fig. 1: Reconstructed image of (a) Algorithm 1, and (b) Algorithm 2 after 6 iterations, using the path matrix calculated based on the MLP.

\begin{tabular}{|c|c|c|c|}
\hline Material & DROP & Robust & Predicted RSP \\
\hline Air (bottom) & 0.064 & 0.064 & 0.0013 \\
\hline Air (top) & 0.076 & 0.075 & 0.0013 \\
\hline PMP & 0.89 & 0.89 & 0.877 \\
\hline LDPE & 1.01 & 1.01 & 0.997 \\
\hline Polystyrene & 1.05 & 1.04 & 1.038 \\
\hline Acrylic & 1.18 & 1.18 & 1.155 \\
\hline Delrin & 1.37 & 1.37 & 1.356 \\
\hline Teflon & 1.80 & 1.80 & 1.828 \\
\hline
\end{tabular}

TABLE I: RSP values of reconstructed images in Figure 1

Figures $2 \mathrm{a}$ and $3 \mathrm{a}$ are between 1 and 5 in size, and the error in RSP of these clusters is about $98 \%$. These artifacts on the boundary of the object should be prevented because of the fact that incorrect RSP values along the boundary of the object affect the accuracy of proton therapy. Based on the images in Figures 2 and 3, and the reconstructed RSP values in Tables II and III, the benefit of the robust technique mentioned in Algorithm 2 is clearly visible, illustrating removal of artifacts from the boundary of the object and generating much more accurate RSP values than DROP itself.

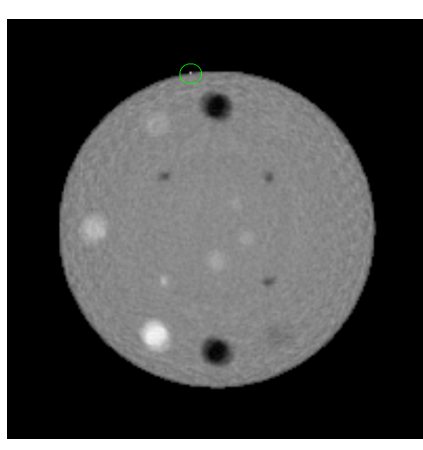

(a) DROP

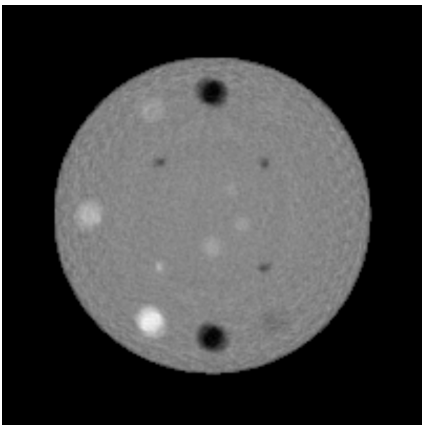

(b) Robust DROP
Fig. 2: Reconstructed image of (a) Algorithm 1, and (b) Algorithm 2 after 12 iterations, using the path matrix calculated based on the MLP and adding Gaussian noise $(\eta=1.0)$ to the elements of the path matrix.

Figures $4 \mathrm{a}$ and $4 \mathrm{~b}$ illustrate the results of the reconstructed images by DROP and Robust respectively, while we have randomly removed 120000 of the histories from the data set. Based on the result, in this case Robust does not necessarily remove all of the artifacts on the boundary of the object, but at least removes a few of them, also based on the RSP values in 


\begin{tabular}{|c|c|c|c|}
\hline Material & DROP & Robust & Predicted RSP \\
\hline Air (bottom) & 0.117 & 0.071 & 0.0013 \\
\hline Air (top) & 0.119 & 0.074 & 0.0013 \\
\hline PMP & 0.919 & 0.872 & 0.877 \\
\hline LDPE & 1.038 & 0.99 & 0.997 \\
\hline Polystyrene & 1.076 & 1.027 & 1.038 \\
\hline Acrylic & 1.211 & 1.161 & 1.155 \\
\hline Delrin & 1.39 & 1.34 & 1.356 \\
\hline Teflon & 1.797 & 1.748 & 1.828 \\
\hline
\end{tabular}

TABLE II: RSP values of reconstructed images in Figure 2

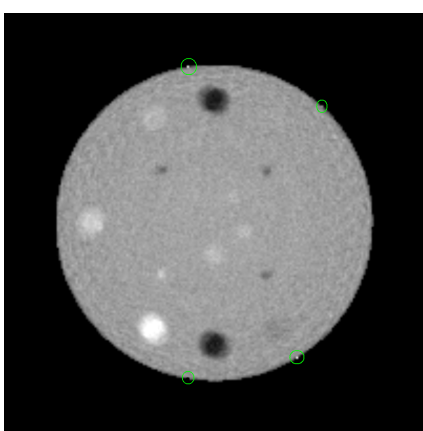

(a) DROP

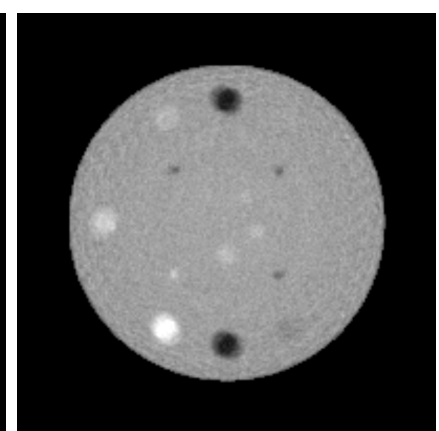

(b) Robust DROP
Fig. 3: Reconstructed image of (a) Algorithm 1, and (b) Algorithm 2 after 12 iterations, using the path matrix calculated based on the MLP and adding Gaussian noise $(\eta=2.0)$ to the elements of the path matrix.

Table IV, Robust generates more accurate RSP values in some of the materials and is never worse than DROP in generating the RSP values for the rest of the materials.

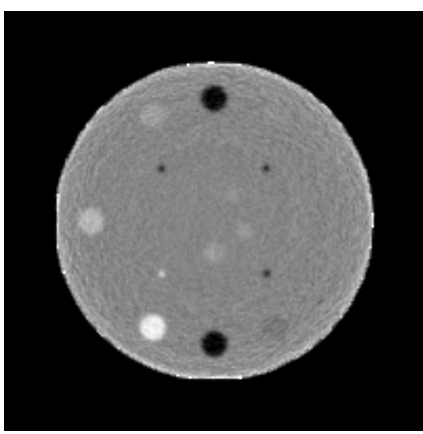

(a) DROP

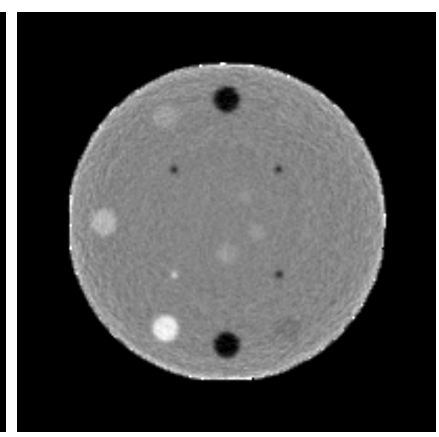

(b) Robust DROP
Fig. 4: Reconstructed image of (a) Algorithm 1, and (b) Algorithm 2 after 6 iterations, while 120000 histories have been removed randomly from the data set.

\begin{tabular}{|c|c|c|c|}
\hline Material & DROP & Robust & Predicted RSP \\
\hline Air (bottom) & 0.25 & 0.21 & 0.0013 \\
\hline Air (top) & 0.24 & 0.2 & 0.0013 \\
\hline PMP & 1.04 & 1.01 & 0.877 \\
\hline LDPE & 1.17 & 1.12 & 0.997 \\
\hline Polystyrene & 1.21 & 1.16 & 1.038 \\
\hline Acrylic & 1.34 & 1.3 & 1.155 \\
\hline Delrin & 1.52 & 1.4 & 1.356 \\
\hline Teflon & 1.92 & 1.9 & 1.828 \\
\hline
\end{tabular}

TABLE III: RSP values of reconstructed images in Figure 3

\begin{tabular}{|c|c|c|c|}
\hline Material & DROP & Robust & Predicted RSP \\
\hline Air (bottom) & 0.044 & 0.044 & 0.0013 \\
\hline Air (top) & 0.057 & 0.056 & 0.0013 \\
\hline PMP & 0.91 & 0.897 & 0.877 \\
\hline LDPE & 1.004 & 1.001 & 0.997 \\
\hline Polystyrene & 1.051 & 1.051 & 1.038 \\
\hline Acrylic & 1.179 & 1.178 & 1.155 \\
\hline Delrin & 1.354 & 1.354 & 1.356 \\
\hline Teflon & 1.781 & 1.781 & 1.828 \\
\hline
\end{tabular}

TABLE IV: RSP values of reconstructed images in Figure 4

\section{CONCLUSION}

Proton CT is a promising imaging modality which could be used in planning proton therapy treatment. In pCT, the goal is to find a good approximation to the linear system of equations of the form $A x=b$. $A$ is an $m \times n$ sparse matrix such that $a_{j}^{i}$ is the intersection length of the $i$ th proton history with the $j$ th voxel element, and $b_{i}$ is an $m \times 1$ vector that contains the water-equivalent path length (WEPL) measurements.

Because of uncertain measurements of the path that a proton takes while traversing the object, and the intersection length of a proton and a voxel, the path matrix $A$ contains some level of noise that causes reconstructing inaccurate RSP values and also generating artifacts on the boundary of the object. In this paper, we have taken the uncertainty associated with the path matrix into account and incorporated robustness into the diagonally-relaxed orthogonal projections algorithm (DROP) to reduce noise associated with the RSP values and artifacts on the object boundary. The images presented here make it clear that the robust approach offers a way to successfully reduce the RSP noise of regions of consistent material inside the object and the noise on the boundary of the object.

In the future, we will investigate a variation of the robust technique by assuming protons follow a cubic spline instead of the computationally expensive MLP technique. Using the the cubic spline as the path that traverses the object is a lot easier than MLP calculations, but at the same time, it is more uncertain. Thus, the robust reconstruction technique holds the potential to generate reconstructed images with the same level of accuracy using cubic splines to fill the cells of the path matrix $A$.

\section{REFERENCES}

[1] V. Bashkirov, R. Schulte, G. Coutrakon, B. Erdelyi, K. Wong, H. Sadrozinski, S. Penfold, A. Rosenfeld, S. McAllister, and K. Schubert, "Development of Proton Computed Tomography for Applications in Proton Therapy," in APPLICATION OF ACCELERATORS IN RESEARCH AND INDUSTRY: Twentieth International Conference, F. D. McDaniel and B. L. Doyle, Eds., vol. AIP Conference Proceedings Volume 1099. Fort Worth (Texas): American Institute of Physics, August 10-15 2008, pp. 460-463, iSBN: 978-0-7354-0633-9.

[2] H.-W. Sadrozinski, V. Bashkirov, B. Colby, G. Coutrakon, B. Erdelyi, D. Fusi, F. Hurley, R. Johnson, S. Kashiguine, S. McAllister, F. MartinezMcKinney, J. Missaghian, M. Scaringella, S. Penfold, V. Rykalin, R. Schulte, K. Schubert, D. Steinberg, and A. Zatserklanyi, "Detector development for proton computed tomography (pct)," in Nuclear Science Symposium and Medical Imaging Conference (NSS/MIC), 2011 IEEE, Oct 2011, pp. 4457-4461.

[3] S. N. Penfold, R. W. Schulte, Y. Censor, V. Bashkirov, S. Macallister, K. E. Schubert, and A. B. Rosenfeld, "Block-iterative and stringaveraging projection algorithms in proton computed tomography image reconstruction," in Biomedical Mathematics: Promising Directions in Imaging, Therapy Planning and Inverse Problems, Y. Censor, M. Jiang, 
and G. Wang, Eds., The Huangguoshu International Interdisciplinary Conference. Madison, WI, USA: Medical Physics Publishing, 2010.

[4] R. Schulte, S. Penfold, J. Tafas, and K. Schubert, "A maximum likelihood proton path formalism for application in proton computed tomography," Med. Phys., vol. 35, pp. 4849-4856, November 2008.

[5] B. Schultze, M. Witt, Y. Censor, K. Schubert, and R. Schulte, "Performance of hull-detection algorithms for proton computed tomography reconstruction," in Infinite Products of Operators and Their Applications, ser. Contemporary Mathematics, S. Reich and A. Zaslavski, Eds., vol. 636. American Mathematical Society, 2015, pp. 211-224.

[6] S. Penfold, A. Rosenfeld, R. Schulte, and K. Schubert, "A more accurate reconstruction system matrix for quantitative proton computed tomography," Med. Phys., vol. 36, no. 10, pp. 4511-4518, October 2009. 